\title{
Effect of External Daylight in Smart Dimmable LED Lighting Systems
}

\author{
Milica Petkovic, Dragana Bajovic, Dejan Vukobratovic, Graeme McCutcheon, Lina Stankovic, Vladimir Stankovic
}

\begin{abstract}
This paper presents an energy-efficient solution for the smart light-emitting diode (LED) lighting system, which simultaneously provides minimal energy consumption and satisfies users' illuminance requirements. Typical office space is illuminated by artificial light received from dimmable LED lamps, as well as daylight coming from external sources, i.e., the sunlight. Besides the possibility to dim LED lamps resulting in reduced LED output power, total illumination intensity at users' location can be managed by reducing overall daylight. The idea to decrease daylight intensity is realized by option to dim the windows in order to properly exploit external lighting. Simulation results show that the proposed algorithm provides greater level of users' satisfaction related to illuminance requirements.

Index Terms-Daylight, dimming, illuminance design, users' requirements, smart LED lighting systems.
\end{abstract}

\section{INTRODUCTION}

In modern society, especially in developed countries, people spend more than $70 \%$ of the daytime in indoor environments, where the illumination is mostly provided by electric lighting [1]. About $20 \%$ of global electric energy usage is exploited for lighting, with tendency to be increased by $40 \%$ in the decades to come [2], [3]. As an efficient way to reduce high energy consumption, smart light-emitting diode (LED) lighting systems have generated research and industrial interests as a modern, persistent, and energy-efficient method of illumination. It is predicted that the smart lighting market will exceed 47 billion $\$$ in next 10 years [4].

Besides savings in energy consumption, the smart LED systems can potentially improve comfort of users. Since most of the time people spend in the offices, it is important to provide a satisfactory level of their comfort during the working hours, in order to avoid underperforming due to inappropriate lighting environment [5]. Still, users' requirements for light are very personal and subjective, and the design of overall illumination in indoor environments can be quite challenging. However, usage of indoor wireless technologies supporting Internet of Things (IoT) applications makes the information

This work has received funding from the European Union Horizon 2020 research and innovation programme under the Marie Skodowska-Curie grant agreement No 734331 .

Milica Petkovic, Dragana Bajovic and Dejan Vukobratovic are with Faculty of Technical Science, Novi Sad, Serbia (e-mails: milica.petkovic@uns.ac.rs dbajovic@uns.ac.rs; dejanv@uns.ac.rs).

Graeme McCutcheon is with Ramboll UK, Glasgow, UK (e-mail Graeme.McCutcheon@ramboll.co.uk).

Lina Stankovic and Vladimir Stankovic are with University of Strathclyde, Department of Electrical and Electronic Engineering, Glasgow, UK (e-mails lina.stankovic@eee.strath.ac.uk; vladimir.stankovic@eee.strath.ac.uk). about occupant preferences, presence and current illumination levels readily available for illumination optimization.

Many studies have focused on reducing energy consumption while satisfying users' requirements in various scenarios [6] [14]. In [6]-[9], maximal energy savings is accomplished by determining the dimming levels of the LED lamps, considering occupants comfort levels at the same time. Additionally, besides artificial light provided by dimmable LED lamps, the open office spaces can be illuminated also by external sources of light, i.e., the sunlight, resulting in daylight illumination at the working plane. Daylight intensity from windows is an important factor in total illumination in open spaces that should also be taken into account. It can be exploited for both energy savings as replacement of artificial light, as well as for better satisfaction of the users' comfort requrements since it is more desirable and health-friendly [10]. In [10]-[14], optimization algorithms in smart LED systems are performed to reduce overall energy consumption while daylight was taken into account.

The aim of this paper is to provide an efficient strategy to reduce total energy consumption of smart LED lighting system implemented in indoor office space. The artificial illumination is controlled by dimming levels of each LED lamp to provide power savings, while daylight contribution is also considered. To the best of authors' knowledge, for the first time, possibility to optimize the intensity of the daylight coming from the windows is included. Commercial tintable smart windows can be employed to reduce and manage the daylight in indoor space [15], [16]. The windows can be shaded by a desired factor, changing the daylight intensity at the users' locations. In that manner, total illuminance contribution can be managed, ensuring more precise satisfaction of the users' requirements for illumination, while simultaneously minimizing total energy consumption of the smart LED system.

\section{System ModeL}

An indoor office environment with rectangular-shaped floor area of dimensions $L_{x} \times L_{y}$ meters and height $h$ meters is considered. Windows can be present, which represent the external source of the light in the room. A smart lighting system with $N$ dimmable ceiling-mounted LED lamps is employed to illuminate the workspace at the floor plane. Each LED lamp consists of $l$ LEDs photodiodes [17]. If $P_{l}$ denotes the transmitted optical power of a single LED, $P_{t}=l P_{l}$ is the maximum transmitted optical power of a LED lamp. The 
positions of $N$ LED sources are fixed and known to all the elements in this system.

The smart LED system output power is related to the dimming vector $\mathbf{s}$ defined as

$$
\mathbf{s}=\left[s_{1}, s_{2}, \ldots, s_{N}\right]^{T}, \quad 0 \leq s_{i} \leq 1, \quad i=1,2, \ldots, N,
$$

where $s_{i}$ represents the dimming level of the $i$-th LED lamp. We assume that $s_{i}=0$ indicates that the $i$-th LED is turned off, while $s_{i}=1$ means that the $i$-th LED radiates at its maximum power $P_{t}$. The output power of the $i$-th LED source is $P_{i}=s_{i} P_{t}$, where we assume $P_{t}$ is the same for all LEDs.

System is designed to provide illumination for a maximum of $M$ users residing in the horizontal workspace plane parallel to the ceiling. Users' positions are known, due to earlier estimation based on some indoor positioning techniques [18], [19]. This assumption is realistic, since we assume the office layout is known as well as position of working desks.

Illumination at the $M$ points on the workspace plane related to the users is denoted as

$$
\mathbf{z}=\left[z_{1}, \ldots, z_{j}, \ldots, z_{M}\right]^{T}, \quad j=1,2, \ldots, M
$$

where $z_{j}$ is the illuminance at the $j$-th user point position.

A simple linear model relates the dimming vector $\mathbf{s}$ and the resulting illumination on the task plane points $\mathbf{z}$ [6]

$$
\mathbf{z}=\mathbf{H} \cdot \mathbf{s}
$$

In the above model, $\mathbf{H}$ represents the illuminance $M \times N$ matrix with elements $h_{j i} \quad(j=1,2, \ldots, M, \quad i=1,2, \ldots, N)$, which denotes the illuminance at the $j$-th point when $s_{i}=1$, i.e., the $i$-th LED is set to maximum output power, while all other LEDs are turned off $\left(s_{j}=0\right.$ for $\left.j \neq i, j=1,2, \ldots, N\right)$.

The above model follows from the light additivity property, and assumes that the dimmable LED lighting system is the only source of light in the room. In general case, the external sources of light (e.g., windows) exist in the room. Illumination due to external sources at the users' positions on the workspace plane is defined as

$$
\mathbf{d}=\left[d_{1}, \ldots, d_{j}, \ldots, d_{M}\right]^{T}, \quad j=1,2, \ldots, M
$$

where $d_{j}$ is the total daylight intensity received at the $j$ th user point position, and is independent on the dimming LED lighting vector. In order to exploit both artificial light and daylight, as well as to ensure better satisfaction of all users' requirements, the windows can be uniformly shaded by a constant factor denoted by $a(0 \leq a \leq 1)$ [15], [16]. When $a=1$, windows are completely 'open' and overall daylight intensity is received at the users' location. When $a=0$, windows are totally shaded, and the daylight will be absent, i.e., $\mathbf{d}=\mathbf{0}$. The model can be easily updated with daylight distribution, thus the resulting illumination at the users' positions is

$$
\mathbf{z}=\mathbf{H} \cdot \mathbf{s}+a \cdot \mathbf{d}
$$

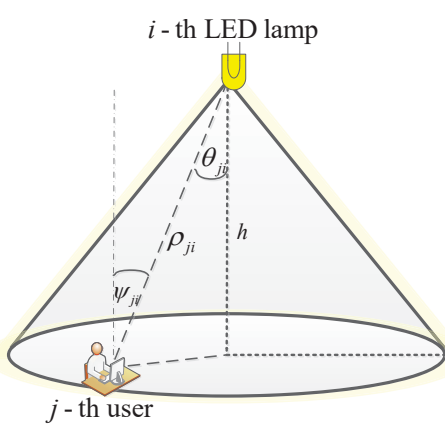

Fig. 1. A LED illumination model.

\section{A. LED illumination model}

The illuminance at the $j$-th user position when the $i$-th LED is fully turned on while all other LEDs are turned off, is defined as [20]-[22]

$$
h_{j i}=\frac{I(0) \cos ^{m_{i}}\left(\theta_{j i}\right)}{\rho_{j i}^{2}} \cos \left(\psi_{j i}\right),
$$

where $I(0)$ denotes the centre luminous intensity of the LED, $\theta_{j i}$ denotes the angle of irradiance with respect to the axis normal to the ceiling, $\psi_{j i}$ represents the angle of incidence with respect to the axis normal to the working plane surface, and $\rho_{j i}$ is the distance between the $i$-th LED and the $j$-th user position (see Fig. 1). It is assumed that $\theta_{j i}=\psi_{j i}$ since the LED and user plane surface are usually parallel. A LED lighting is described by a Lambertian radiation pattern with the order $m_{i}$ defined as $m_{i}=-\ln 2 / \ln \left(\cos \Phi_{1 / 2, i}\right)$, where the semi-angle at the half-illuminance of the $i$-th LED is denoted by $\Phi_{1 / 2, i}$. It is assumed that all LED lamps are characterized by the same semi-angle at the half-illuminance, i.e., $\Phi_{1 / 2, i}=$ $\Phi_{1 / 2}$ and $m_{i}=m$ for all $i=, 1, \cdots, N$.

\section{PROBLEM FORMULATION}

The main task of smart LED illumination system is to design illumination vector $\mathbf{z}$ based on users' requirements by selecting optimized dimming vector $\mathbf{s}$ and shading coefficient $a$. The illumination vector $\mathbf{z}$ clearly depends on definition of user requirements, which should be satisfied. It is assumed that every user requires certain level of illumination, thus the vector of desired illuminance requirements for $M$ users is defined as

$$
\mathbf{r}=\left[r_{1}, \ldots, r_{j}, \ldots, r_{M}\right]^{T}, \quad j=1,2, \ldots, M .
$$

Furthermore, it is adopted that every user requires minimal illumination, i.e., that $z_{j} \geq l_{j}$, where $l_{j}$ is the lower value of illumination requirement of the $j$-th user. The vector of minimal illuminance requirements for $M$ users is defined as

$$
\mathbf{l}=\left[l_{1}, \ldots, l_{j}, \ldots l_{M}\right]^{T}, \quad j=1,2, \ldots, M,
$$

As previously stated, besides influencing illumination, the dimming vector $\mathbf{s}$ is directly related to energy consumption; namely, total system output power is $P_{t} \cdot \sum_{i=1}^{N} s_{i}$. Thus, energy consumption is directly and linearly related to the $\ell_{1}$-norm of vector s, i.e., $\|\mathbf{s}\|_{1}=\sum_{i=1}^{N} s_{i}$. We can define the energy 
saving factor $G$ as a ratio between the total consumed power and the total system output power when all LEDs are fully turned on as

$$
G=\frac{P_{t}\|\mathbf{s}\|_{1}}{P_{t} N}=\frac{\|\mathbf{s}\|_{1}}{N}
$$

Clearly, favouring solutions with smaller $\|\mathbf{s}\|_{1}$, i.e., the ones with lower $G$, leads to reduced energy consumption. Additionally, in order to manage overall illumination through the open office space, the constant $a$ can be also optimized, reducing the daylight distribution density over the workspace.

The formulated optimization problem for considered smart LED scenario aims to optimize dimming vector $\mathbf{s}$ and shading coefficient $a$. It is formulated as

$$
\begin{array}{cc}
\underset{\mathbf{s}, a}{\operatorname{minimize}} & \|\mathbf{s}\|_{1}+\lambda\|\mathbf{H} \mathbf{s}+a \mathbf{d}-\mathbf{r}\| \\
\text { subject to } & \mathbf{H s}+a \mathbf{d} \geq \mathbf{l} \\
& 0 \leq \mathbf{s} \leq 1, \mathbf{s} \in \mathbb{R}^{N} \\
& 0 \leq a \leq 1, a \in \mathbb{R}
\end{array}
$$

where $\mathbf{H}$ is given by (6) and parameter $\lambda(0 \leq \lambda \leq 1)$ sets the priority by tuning the relative importance of the minimizing energy consumption and satisfying users' requirements. To solve the optimization problem in (10), we can employ CVX, a package for specifying and solving convex programs [23].

\section{NUMERICAL EXAMPLE}

In this section some examples of considered scenario are presented, when the proposed algorithm is applied to a typical office space with dimensions $L_{x} \times L_{y} \times h=10 \times 10 \times 3$ meters. There are $N=9$ dimmable ceiling-mounted LED lamps arranged as an $N_{x} \times N_{y}=3 \times 3$ array. It is assumed that each LED lamp consists of $l=100$ LEDs, arranged as an $10 \times 10$ array. Furthermore, we consider the following model for LED output power, derived from the LED example in the literature [20]. Input voltage of LED and input current are $6.42 \mathrm{~V}$ and $700 \mathrm{~mA}$, respectively. Thus, the electrical power is set to $P_{e}=4.494 \mathrm{~W}$. The electrical/optical conversion efficiency takes a value 0.101 . The optical output power of each LED is $P_{l}=0.452 \mathrm{~W}$, total luminous flux is
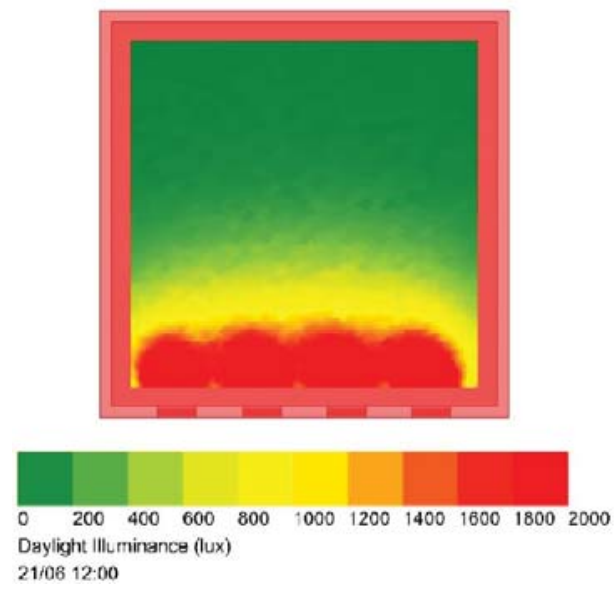

Fig. 2. Daylight intensity in the considered office.

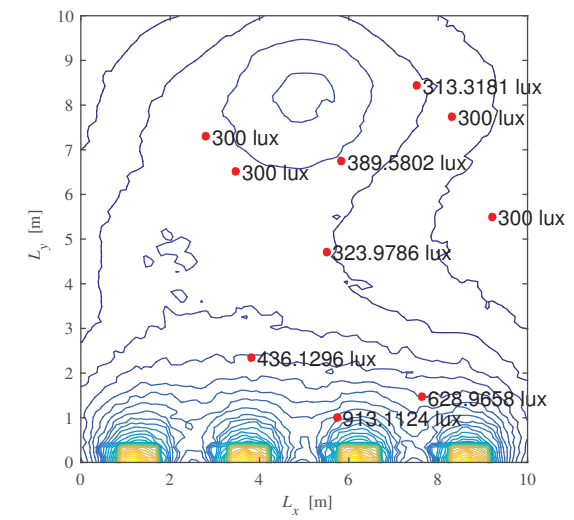

Fig. 3. Illumination distribution, $M=10, \lambda=0.01, \Phi_{1 / 2}=60^{0}$.

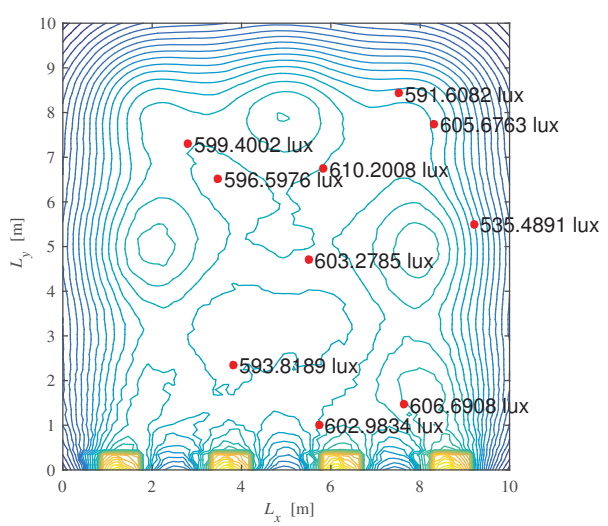

Fig. 4. Illumination distribution, $M=10, \lambda=0.5, \Phi_{1 / 2}=60^{0}$.

$\Phi=107.16 \mathrm{~lm}$. Based on $\Phi=2 \pi I(0) /(1+m)$, the centre luminous intensity of LED for different values of the semiangle at the half-illuminance can be determined. For example, if $\Phi_{1 / 2}=20^{0}$ and $\Phi_{1 / 2}=60^{\circ}$, it holds $I(0)=207.11 \mathrm{~cd}$ and $I(0)=34.11 \mathrm{~cd}$, respectively.

Our example of the office space assumes four windows each with dimensions $1 \times 2$ meters. By employing light sensor devices, in the absence of artificial LED lights, measurements can be performed during various periods in order to determine the level of daylight illuminance at the working horizontal plane. The strongest daylight intensity (in the noon) is measured and presented in Fig. 2.

In following examples, for each scenario we assume a priori knowledge of $M$ users location and illuminance requirements, i.e., $r_{j}=600$ and $l_{j}=300$ lux for $j=1,2, \ldots, M$. Unless otherwise is stated, the semi-angle at the half-illuminance is $\Phi_{1 / 2}=60^{\circ}$, and the priority parameter is set to be $\lambda=0.01$ since our primary aim is to provide energy savings.

Figs. 3 and 4 depict the illumination distribution for the scenario when $M=10$ users with known positions are placed randomly in the office, while the priority parameter is 0.01 and 0.5 , respectively. In Fig. 3, the primary task is to reduce 


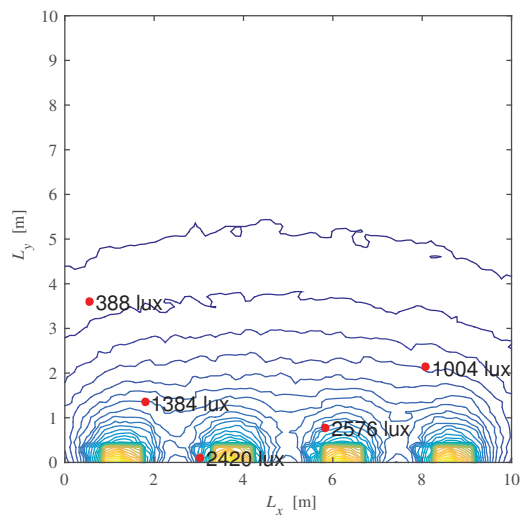

Fig. 5. Illumination distribution, $a=1, M=5, \lambda=0.01, \Phi_{1 / 2}=60^{0}$.

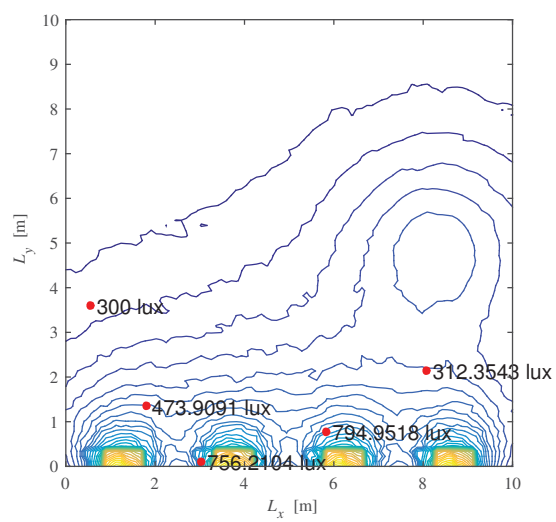

Fig. 6. Illumination distribution, $M=5, \lambda=0.01, \Phi_{1 / 2}=60^{0}$.

total energy consumption, thus the priority parameter is set to be $\lambda=0.01$. Five of nine LEDs are turned on and dimmed in order to provide a certain level of illumination at the users' positions, ensuring maximal possible energy savings. The energy saving factor is $G=0.2602$. Since some of users are placed near the windows, but some at the opposite side of the room, the optimized value of the shading coefficient equals to $a=0.4196$. The shading coefficient is optimized in order to reduce daylight in that way to achieve minimal lighting near the windows and simultaneously providing enough daylight intensity to very distant users. The same scenario is presented in Fig. 4, while the priority parameter is $\lambda=0.5$, meaning that the more important task is to satisfy users' requirements compared to energy savings. Now, the energy saving factor is $G=0.8648$ (even four of nine LEDs are fully turned on) and the optimized shading coefficient is $a=0.0961$. Comparing the results in Figs. 3 and 4, it is obvious that generated illumination in Fig. 4 requires more power waste since $G$ is quite greater, but the level of illumination on users' position is pretty close to the desired one, thus the users' satisfaction is ensured in larger extent.

Figs. 5 and 6 present designed illumination distribution

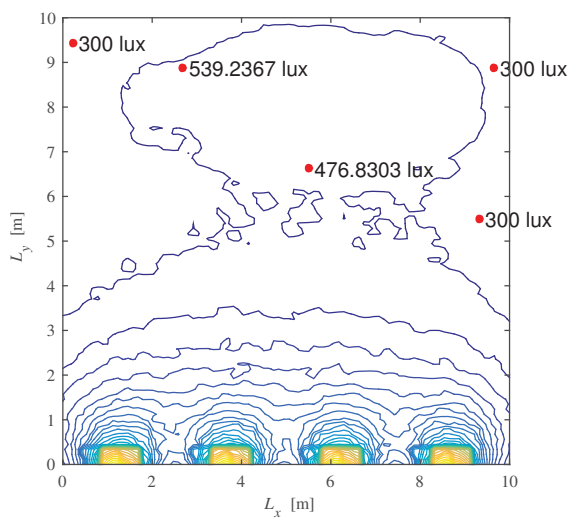

Fig. 7. Illumination distribution, $a=1, M=5, \lambda=0.01, \Phi_{1 / 2}=60^{0}$.

by using proposed algorithm, with assumption that the users are placed near windows and external daylight significantly impacts the illumination at their locations. Result presented in Fig. 5 is obtained under assumption that the windows are unshaded, i.e., $a=1$. After performing the optimization algorithm, the dimming vector equals to zero, i.e., all LEDs are turned off and users at given positions are illuminated only by daylight. The energy saving factor is than $G=0$ and power saving is maximal possible. Still, from Fig. 5 it is obvious that the users' requirements for illumination are not fully satisfied. More precisely, four of five users are illuminated with notably greater amount of light intensity than desired (two even four times more than defined desired requirement). On the other hand, Fig. 6 depicts generated illumination for the same system, when daylight intensity can be reduced by shading the windows. After performing the algorithm, the energy saving factor is obtained to be $G=0.0981$. It is apparent that requirements of users are met in greater extent than in Fig. 5. By introducing the possibility to shade the windows in Fig. 6, the external light is reduced by an optimized factor $a=0.3032$. Although one of nine LEDs is radiating and a certain energy consumption exists, significant improvement of users' satisfaction is achieved.

Designed illumination distribution for the same scenario is shown in Fig. 7 assuming that the users are placed randomly but constrained only to the half of the room opposite of the windows. Since the external daylight will have a minor influence at their locations, the windows will not be shaded at all $(a=1)$. The energy saving factor is $G=0.3240$ since the LEDs must be turned on with a certain dimming level in order to contribute to the daylight which has very low intensity at the locations opposite of the windows. Illumination on users' locations is quite close to desired and not lower than minimal illumination requirements, which results in great users' satisfaction.

Finally, illumination distribution of the same room example with windows shading is observed and presented in Figs. 8 and 9. The semi-angle at the half-illuminance is $\Phi_{1 / 2}=20^{\circ}$, 


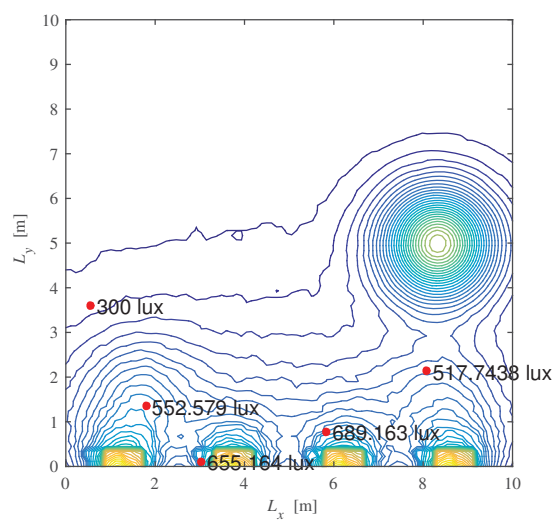

Fig. 8. Illumination distribution, $a=1, M=5, \lambda=0.01, \Phi_{1 / 2}=20^{0}$.

thus the LED lamp radiates very narrow optical beam and the circular area on the floor covered by a single LED lamp is significantly smaller compared with previous examples. Fig. 8 depicts the example when users are placed near windows. Compared to Fig. 6 where $\Phi_{1 / 2}=60^{0}$ when generated algorithm gives $G=0.0981$, similar energy saving is obtained with lower semi-angle in Fig. 8 since $G=0.1024$. Observing Figs. 6 and 8, similar users' satisfaction is provided in Fig. 8 with focused LED beams. Fig. 9 shows the same scenario when users are located opposite of the windows. The power consumption equals to $G=0.2109$ and it is lower than in Fig. 7 when $\Phi_{1 / 2}=60^{\circ}$. With narrower LED beams improved power saving can be achieved providing similar satisfaction of users' illumination requirements.

\section{CONCLUSION}

An energy-efficient strategy for the smart lighting system has been proposed, considering the LED lamps have possibility to be dimmed. External sources of light have been taken into consideration, while the level of daylight can be managed by windows shading. An optimization problem is solved in order to provide minimal power consumption and to ensure illuminance requirements for the users in the office. It has been concluded that possibility to manage intensity of the daylight makes generated illuminance energy efficient while satisfying users' requirements in a great extent.

\section{REFERENCES}

[1] S. Brasche and B. Wolfgang, "Daily time spent indoors in German homesbaseline data for the assessment of indoor exposure of German occupants," Int. J. Hyg. Environ. - Health, vol. 208, no. 4, pp. 247-253, 2005.

[2] A. Bahga and V. Madisetti, Internet of Things: A Hands-On Approach, Vpt., 2015.

[3] P. Waide, S. Tanishima, and P. Harrington Lights Labours Lost: Policies for Energy-efficient Lighting, OECD/IEA Publishing, 2006.

[4] Smart Lighting: A Global Strategic Business Report, Global Industry Analysts Inc. 2015

[5] A. Steidle, L. Werth, Jd Boe and K. Sedlbauer, "How to create sustainable lighting for users? Psychological mechanisms underlying lighting effects," in Proceedings Experiencing light 2014: international conference on the effects of light on wellbeing, Eindhoven, Netherlands, 10-11 November, 2014, pp. 78-81.

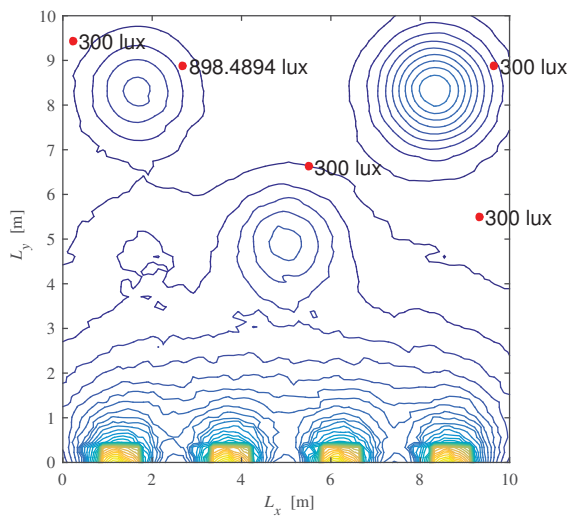

Fig. 9. Illumination distribution, $M=5, \lambda=0.01, \Phi_{1 / 2}=20^{0}$.

[6] X. Wang and J.-PMG. Linnartz, "Intelligent illuminance control in a dimmable LED lighting system," Lighting Research \& Technology, vol. 49, no. 5, pp. 603-617, 2017.

[7] D. Caicedo, A. Pandharipande, and G. Leus, "Occupancy-based illumination control of LED lighting systems," Lighting Res. Technol. vol. 43, pp. 217-234, 2011.

[8] J. Dong and A. Pandharipande, "Efficient distributed control of lightemitting diode array lighting systems," Opt. Letters, vol. 37, no. 14, pp. 2910-2912, 2012.

[9] S. H. Lee and J. K. Kwon. "Distributed dimming control for LED lighting," Opt. express, vol. 21, no. 106, pp. A917-A932, 2013.

[10] V. Singhvi, A. Krause, C. Guestrin, J. Garrett, and H. S. Matthews, "Intelligent light control using sensor networks," in Proc. of ACM Conference on Embedded Networked Sensor Systems, 2005.

[11] A. Peruffo, A. Pandharipandea, D. Caicedo, and L. Schenato, "Lighting control with distributed wireless sensing and actuation for daylight and occupancy adaptation," Energy and Buildings, vol. 97, pp. 13-20, 2015.

[12] N. van de Meugheuvel, et al. "Distributed lighting control with daylight and occupancy adaptation," Energy and Buildings, vol. 75, pp. 321-329, 2014.

[13] S. Yoo, et al. "A sensor-less LED dimming system based on daylight harvesting with BIPV systems," Opt. express, vol. 22, pp. A132-A143, 2014.

[14] D. Caicedo and A. Pandharipande, "Distributed illumination control with local sensing and actuation in networked lighting systems," IEEE Sens. J., vol. 13, no.3, pp. 1092-1104, 2011.

[15] R. Baetens, B. P. Jelle, A. Gustavsen, "Properties, requirements and possibilities of smart windows for dynamic daylight and solar energy control in buildings: A state-of-the-art review," Solar Energy Materials and Solar Cells. vol. 94, no. 2, pp. 87105, 2010.

[16] J. McGrath, "Cooling down the house: A new smart glass could block both heat and light", Digital Trends, Aug. 2015, Retrieved Aug. 2018.

[17] D. A. Basnayaka and H. Haas, "Design and analysis of a hybrid radio frequency and visible light communication system," IEEE Trans. Commun., vol. 65, no. 10, pp. 4334 - 4347, Oct. 2017.

[18] Y. Gu, A. L. Yanying, and I. Niemegeers, "A survey of indoor positioning systems for wireless personal networks," IEEE Commun. Surv. Tutor., vol. 11, no. 1, pp. 13-32, 2009.

[19] T-H. Do and M. Yoo, "An in-depth survey of visible light communication based positioning systems," Sensors, vol. 16, no. 5, 2016.

[20] T. Komine, Visible Light Wireless Communications and Its Fundamental Study, Keio University, PhD thesis, Japan, 2005

[21] T. Komine and M. Nakagawa, "Fundamental analysis for visible-ligh communication system using LED lights," IEEE Trans. Consum. Electron., vol. 50, no. 1, pp. 100-107, Feb. 2004.

[22] Z. Ghassemlooy, W. Popoola, and S. Rajbhandari, Optical Wireless Communications: System and Channel Modelling With MATLAB. Boca Raton, FL, USA: CRC Press, 2013.

[23] M. Grant and S. Boyd, CVX: Matlab software for disciplined convex programming, version 2.0 beta. http://cvxr.com/cvx, Sept. 2013. 